\title{
Accidental degeneracy in a simple quantum system: A new symmetry group for a particle in an impenetrable square-well potential
}

\section{F. Leyvraz}

Instituto de Física, Laboratorio de Cuernavaca, UNAM, Apdo. Postal 139-B, Cuernavaca, Morelos, Mexico

\author{
A. Frank \\ Instituto de Física, Laboratorio de Cuernavaca, UNAM, Apdo. Postal 139-B, Cuernavaca, Morelos, Mexico \\ and Instituto de Ciencias Nucleares, UNAM, Apdo. Postal 70-543, Circuito Exterior, C. U. 04510 \\ Mexico, D. F., Mexico \\ R. Lemus \\ Instituto de Ciencias Nucleares, UNAM, Apdo. Postal 70-543, Circuito Exterior, C. U. 04510 Mexico, \\ D. F., Mexico \\ M. V. Andrés \\ Departamento de Física Atómica, Molecular y Nuclear Facultad de Física, Universidad de Sevilla, \\ Apdo. 1065, 41080 Sevilla, Spain
}

(Received 2 December 1996; accepted 30 April 1997)

\begin{abstract}
The two-dimensional square-well potential is one of the simplest quantum-mechanical systems that exhibits accidental degeneracy. We show that the double degeneracy present is a consequence of a dynamical symmetry and derive a new symmetry group associated with the system. (C) 1997
\end{abstract} American Association of Physics Teachers.

\section{INTRODUCTION}

Accidental degeneracy in quantum systems usually signals the presence of a hidden symmetry group for which the degeneracy is rendered normal, that is, in terms of which the observed state degeneracies correspond to the dimensions of the irreducible representations of the group. ${ }^{1}$ This is the case for the nonrelativistic hydrogenic atom, where the $S O(4)$ symmetry explains the angular momentum independence of the energy spectrum. ${ }^{2}$ Many other physical systems displaying larger degeneracies than anticipated have been studied, and in most instances the corresponding hidden symmetries have been identified. In most cases, however, this requires a very careful and detailed analysis, which has prompted the authors of Ref. 3 to compare it more with art than with science. Moreover, these symmetries usually correspond to continuous transformations and the corresponding symmetry groups are thus Lie groups or supergroups. ${ }^{4}$ It may come as a surprise that one of the simplest and most studied systems in introductory quantum mechanics, that of the free particle in an impenetrable square box, exhibits accidental degeneracy and that, to our knowledge, no group-theoretical explanation has been provided in the literature. The problem is discussed in many quantum mechanics textbooks. For example, Liboff assigns the degeneracy to the $x \leftrightarrow y$ symmetry transformation between wave functions, but does not discuss either the symmetry classification of the states or the distinction between normal and accidental degeneracies in the system. ${ }^{5}$ Other books do analyze the geometrical symmetries in the problem, but fail to explain the presence of accidental degeneracy. ${ }^{6}$ In contrast to other hidden symmetry analyses that have been carried out, a distinguishing feature of this system (and that of its three-dimensional extension) is that the patent geometrical symmetry of the box corresponds to a point group and not to a continuous set of transformations. Perhaps for this reason the problem has not been studied using the traditional methods, ${ }^{3}$ since its analysis requires a combination of discrete and continuous group techniques. In this article we re-examine the two-dimensional particle in a box and derive, using group-theoretical arguments, the dynamical symmetry responsible for the observed double degeneracy. We also construct a new symmetry group which arises from the combination of this (continuous) symmetry and the eight discrete operations of the square-well geometric symmetry group. The new group is similar to the kind of space groups common in solid state physics. We then proceed to show explicitly that the group explains the observed double degeneracy of the system.

\section{THE SQUARE-WELL POTENTIAL}

A free particle enclosed by an impenetrable twodimensional square box of side $L$ has the eigenstates

$$
\psi_{n_{1} n_{2}}(x, y)=\frac{2}{L} \sin \left(\frac{n_{1} \pi x}{L}\right) \sin \left(\frac{n_{2} \pi y}{L}\right),
$$

where $n_{1}, n_{2}$ are positive integers. These states satisfy the condition $\psi_{n_{1} n_{2}}=0$ at the boundaries of the box. In Fig. 1 we indicate the coordinate system selected for the states (1). It is in this system that the eigenfunctions take the simple form (1). The corresponding eigenvalues are given by

$$
E_{n_{1}, n_{2}}=\frac{\hbar^{2} \pi^{2}}{2 \mu L^{2}}\left(n_{1}^{2}+n_{2}^{2}\right) .
$$

We can readily see that there are two kinds of degeneracies present. The first kind, which is the one we shall consider in this paper, is a double degeneracy that occurs whenever $n_{1}$ $\neq n_{2}$, since by interchanging the $n_{1}$ and $n_{2}$ labels in (1), we find

$$
E_{n_{1}, n_{2}}=E_{n_{2}, n_{1}} \text {. }
$$

The second kind of degeneracy is more subtle and occurs when we have the following kind of "Pythagorean" relations:

$$
n_{1}^{2}+n_{2}^{2}=n_{3}^{2}+n_{4}^{2} \quad \text { with } n_{i} \neq n_{j} \text { for all } i, j
$$




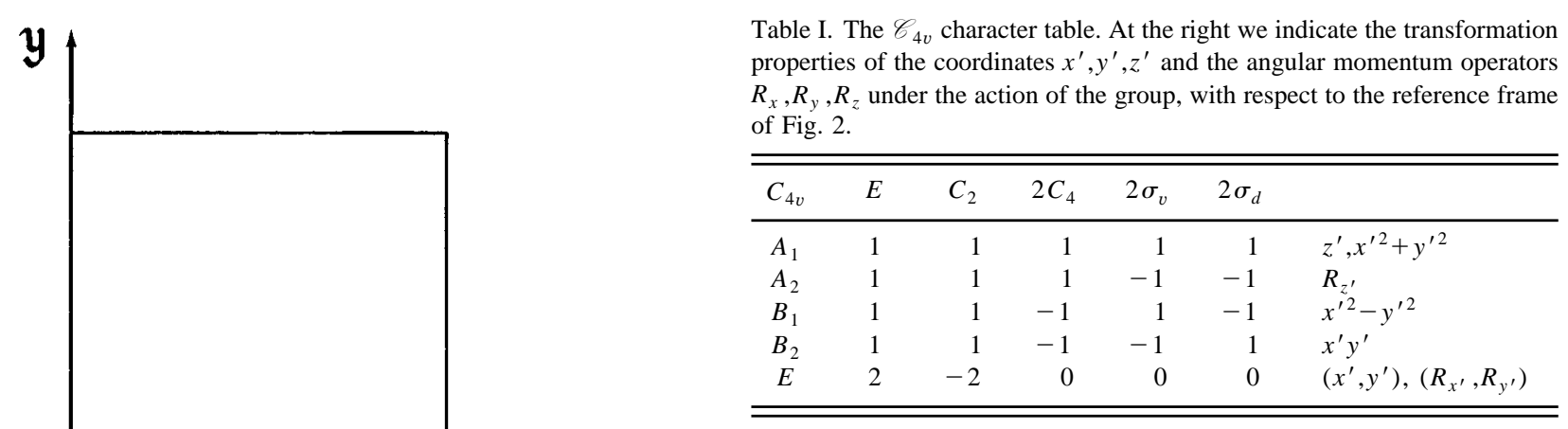

Fig. 1. Selected (unprimed) coordinate system for the square-well potential, with origin at a corner of the box.

and higher order relations of this sort, i.e., the equality of three or more of these sums of squares. The full degeneracy of the problem, including the ones in (4), has been discussed by Wai-Kee $\mathrm{Li}$, giving rise to a formula which arises from the Gaussian factorization theorem. This result is useful to evaluate the degree of degeneracy as the quantum numbers grow. ${ }^{7}$ We shall not attempt to explain the existence of the "Pythagorean' degeneracy (4) in terms of a larger symmetry group in this paper, so from our point of view they will remain "accidental." It is, of course, an interesting question whether they can also be understood in this way, a matter which probably involves the establishment of some kind of new connection between group-theoretical methods and number theory. The analysis of the double degeneracies (3), however, may shed some light on this question.

Returning to Eq. (3), we shall now start by showing that the apparent symmetry of the system is unable to account for this behavior. What is the explicit symmetry group associated with the particle in the square box? The system is clearly invariant under all operations that transform the square box onto itself, i.e., the $\mathscr{C}_{4 v}$ point group of powers of $\pi / 4$ rotations and reflections. ${ }^{8}$ These operations are indicated in Fig. 2, while in Table I we write down the $\mathscr{C}_{4 v}$-character table. At the right of Table I we indicate the way that the coordinates and angular momentum operators transform under the group. We see from Table I that this group has five kinds of irreducible representations (I.R.), four of them one

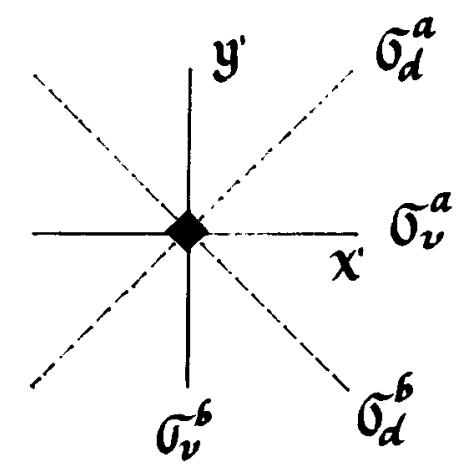

Fig. 2. Primed coordinate system for symmetry elements of the group $C_{4 v}$, with origin at the center of the box.

dimensional and a single two-dimensional one. In order for this group to explain the degeneracy (3) of the system, the set of doubly degenerate states $\psi_{n_{1} n_{2}}$ and $\psi_{n_{2}, n_{1}}$, with $n_{1} \neq n_{2}$, should transform as the twodimensional irreducible representation, $E$. We shall prove, however, that this is not the case. To this end, we define the linear combinations

$$
\phi_{n_{1} n_{2}}^{ \pm}=\frac{1}{\sqrt{2\left(1+\delta_{n_{1} n_{2}}\right)}}\left(\psi_{n_{1} n_{2}} \pm \psi_{n_{2} n_{1}}\right),
$$

which have the same energy spectrum as the states (1) and are more appropriate, since they carry the I.R. of $\mathscr{C}_{4 v}$, as we now show by applying to them the operators of the group. It is enough to consider a single operator in each $\mathscr{C}_{4 v}$ class, from which we find

$$
\begin{aligned}
& \hat{E} \phi_{n_{1} n_{2}}^{ \pm}=\phi_{n_{1} n_{2}}^{ \pm}, \\
& \hat{C}_{2} \phi_{n_{1} n_{2}}^{ \pm}=(-)^{n_{1}+n_{2}} \phi_{n_{1} n_{2}}^{ \pm}, \\
& \hat{C}_{4} \phi_{n_{1} n_{2}}^{ \pm}=\alpha_{n_{1} n_{2}}^{ \pm} \phi_{n_{1} n_{2}}^{+}+\beta_{n_{1} n_{2}}^{ \pm} \phi_{n_{1} n_{2}}^{-}, \\
& \sigma_{v}^{a} \phi_{n_{1} n_{2}}^{ \pm}=\alpha_{n_{1} n_{2}}^{ \pm} \phi_{n_{1} n_{2}}^{+}-\beta_{n_{1} n_{2}}^{ \pm} \phi_{n_{1} n_{2}}^{-}, \\
& \sigma_{d}^{a} \phi_{n_{1} n_{2}}^{ \pm}= \pm \phi_{n_{1} n_{2}}^{ \pm},
\end{aligned}
$$

where

$$
\begin{aligned}
& \alpha_{n_{1} n_{2}}^{ \pm}=\frac{1}{2}\left((-)^{n_{2}+1} \pm(-)^{n_{1}+1}\right), \\
& \beta_{n_{1} n_{2}}^{ \pm}=\frac{1}{2}\left((-)^{n_{2}} \pm(-)^{n_{1}+1}\right) .
\end{aligned}
$$

The operations considered in (6) are indicated in Fig. 2 and the result can be simply deduced from the action of the group operators on the coordinates $(x, y)$ and substituting in the $\psi$ 's in (1) and the $\phi$ 's in (5). For example, $\hat{C}_{2} x=L-x, \hat{C}_{2} y$ $=L-y$, and the result (6b) can be found by direct substitution in the wave functions.

It is important to emphasize that in order to carry out the symmetry analysis of the system it is necessary to use the states (5) and not the wave functions (1), since the latter, in general, do not transform irreducibly under the $\mathscr{C}_{4 v}$ group, but rather as a linear combination of two different representations. This is an important point, which is often a source of confusion in quantum mechanics textbooks. For example, the $\hat{\sigma}_{d}^{a}$ operator in (6e), while clearly connecting the states $\psi_{n_{1} n_{2}}$ and $\psi_{n_{2} n_{1}}$, does not mix the states (5) among themselves. The mixing of the $\psi$ 's, while implying a degeneracy, does not provide a reason for its occurrence. 
We now return to our discussion. From relations (6) and the character Table I we can identify the symmetry character of the $\phi_{n_{1}, n_{2}}^{ \pm}$according to the parity of the labels. For example, if $n_{1}=2 k, n_{2}=2 l$ ( $k, l$ integers), we find

$$
\alpha_{2 k, 2 l}^{+}=-1, \quad \alpha_{2 k, 2 l}^{-}=0, \quad \beta_{2 k, 2 l}^{+}=0, \quad \beta_{2 k, 2 l}^{-}=1
$$

and from (6):

$$
\hat{E} \phi_{2 k, 2 l}^{ \pm}=\phi_{2 k, 2 l}^{ \pm}, \quad \hat{C}_{2} \phi_{2 k, 2 l}^{ \pm}=\phi_{2 k, 2 l}^{ \pm}, \quad \hat{C}_{4} \phi_{2 k, 2 l}^{ \pm}=\mp \phi_{2 k, 2 l}^{ \pm},
$$

$\hat{\sigma}_{v}^{a} \phi_{2 k, 2 l}^{ \pm}=-\phi_{2 k, 2 l}^{ \pm}, \quad \hat{\sigma}_{d}^{a} \phi_{2 k, 2 l}^{ \pm}=\phi_{2 k, 2 l}^{ \pm}$.

We see that $\phi_{2 k, 2 l}^{+}$and $\phi_{2 k, 2 l}^{-}$are not mixed by the group operators and, furthermore, by comparing (8b) with Table I we find that

$$
\phi_{2 k, 2 l}^{+} \rightarrow B_{2}, \quad \phi_{2 k, 2 l}^{-} \rightarrow A_{2} \quad(l \neq k)
$$

is their symmetry character. Note that due to (5) $\phi_{n, n}^{-}=0$ and only $\phi_{2 k, 2 k}^{+}$survives, but still transforms as $B_{2}$. Doing the same analysis for $n_{1}=2 k+1, n_{2}=2 l+1$, we find

$$
\phi_{2 k+1,2 l+1}^{+} \rightarrow A_{1}, \quad \phi_{2 k+1,2 l+1}^{-} \rightarrow B_{1} \quad(l \neq k) .
$$

Again, for $l=k$ the $A_{1}$ state $\phi_{2 k+1,2 k+1}^{+}$is nondegenerate. Finally, for $(-)^{n_{1}} \neq(-)^{n_{2}}$, we find that $\phi_{n_{1} n_{2}}^{+}$and $\phi_{n_{1} n_{2}}^{-}$do mix, since, e.g., from (6) and (7)

$$
\hat{C}_{4} \phi_{2 k, 2 l+1}^{+}=\phi_{2 k, 2 l+1}^{-} \text {. }
$$

We conclude that the pair of states $\phi_{n_{1}, n_{2}}^{ \pm}, n_{1} \neq n_{2}$, carry the double-valued I.R. $E$ only when $(-)^{n_{1}} \neq(-)^{n_{2}}$. For this case the degeneracy (3) is indeed explained by $\mathscr{C}_{4 v}$. However, in the case when $n_{1} \neq n_{2}$ and the parities are the same, for both even and odd values of the quantum numbers the set of degenerate states $\phi_{n_{1} n_{2}}^{+}$and $\phi_{n_{1} n_{2}}^{-}$carry different onedimensional I.R. of $\mathscr{C}_{4 v}$ and are thus "accidentally" degenerate under the action of this group. From the point of view of $\mathscr{C}_{4 v}$ there is no reason for $\left(B_{2}, A_{2}\right)$ or $\left(A_{1}, B_{1}\right)$ states to be degenerate. In Figs. 3 and 4 we display particular $\phi_{n_{1}, n_{2}}^{ \pm}$ wave functions associated with $E$ and $\left(B_{2}, A_{2}\right)$ symmetries, respectively. Note that no obvious geometric operation can transform the $\phi^{+}$into the $\phi^{-}$for the latter case.

\section{HIDDEN SYMMETRY}

To attempt an explanation for this behavior we now search for additional operators that can mix the states (9) and (10) among themselves and thus should lie outside $\mathscr{C}_{4 v}$. To this end, we remind the reader that in the case of the nonrelativistic hydrogenic atom a similar situation occurs, and the solution stems from the existence of a nongeometrical symmetry arising from the particular (Coulomb) potential involved. ${ }^{2}$ This is a so-called "dynamical symmetry,' expressed through the conservation of the Runge-Lenz vector $\mathbf{A}$ and through the closure (at fixed energy) of the commutation relations involving $\mathbf{A}$ and the angular momentum $\mathbf{L}$, corresponding to an $S O(4)$ symmetry. ${ }^{2}$ In the square-box system, however, there is no potential inside the box while there is an infinite potential at the boundary, and it is the shape of the box that entirely imposes the $\mathscr{C}_{4 v}$ symmetry. Since the eigenstates (5) satisfy the appropriate boundary conditions, the symmetry group for the system can be more simply de-

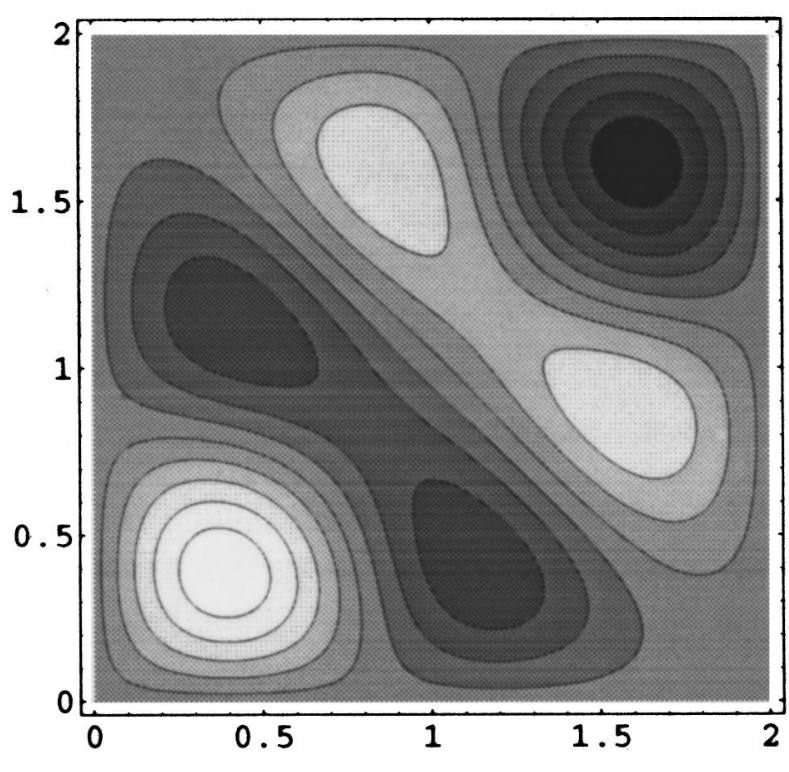

(a)

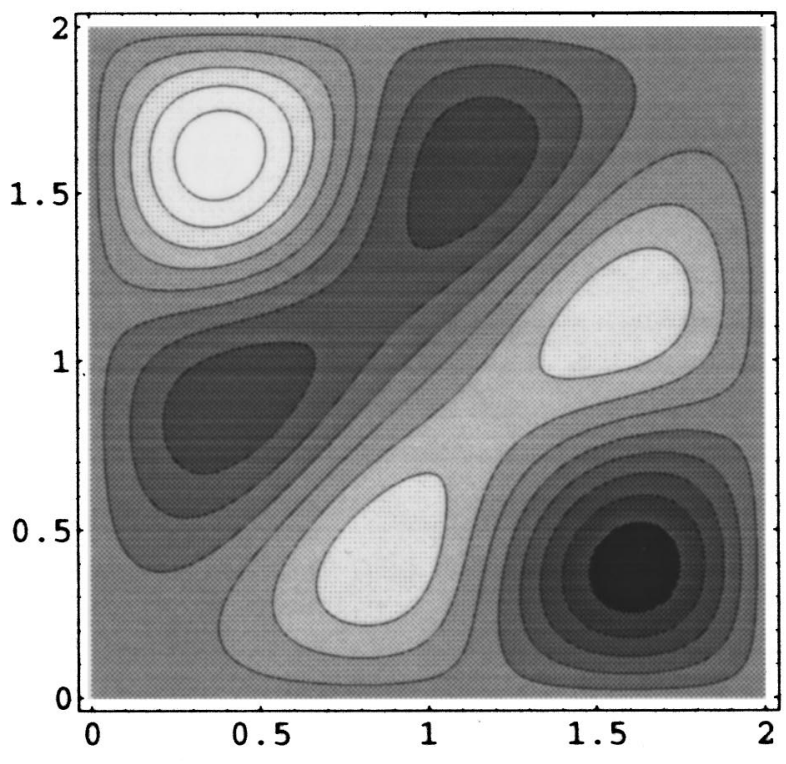

(b)

Fig. 3. Contour plot for the wave function (a) $\phi_{23}^{+}$and (b) $\phi_{23}^{-}$.

fined through the following requirements. The symmetry operations $\hat{g}_{i}$ should commute with the particle's Hamiltonian inside the box:

$$
\hat{H}=-\frac{\hbar^{2}}{2 \mu}\left(\frac{\partial^{2}}{\partial x^{2}}+\frac{\partial^{2}}{\partial y^{2}}\right),
$$

i.e.,

$$
\left[\hat{g}_{i}, \hat{H}\right]=0,
$$

and, in addition, when acting on the eigenstates (5) the $\hat{g}_{i}$ should lead to states with the same boundary conditions. An arbitrary $O(2)$ transformation (two-dimensional rotations and reflections) satisfies (13), but only its $\mathscr{C}_{4 v}$ subgroup operations preserve the boundary conditions. By imposing the 


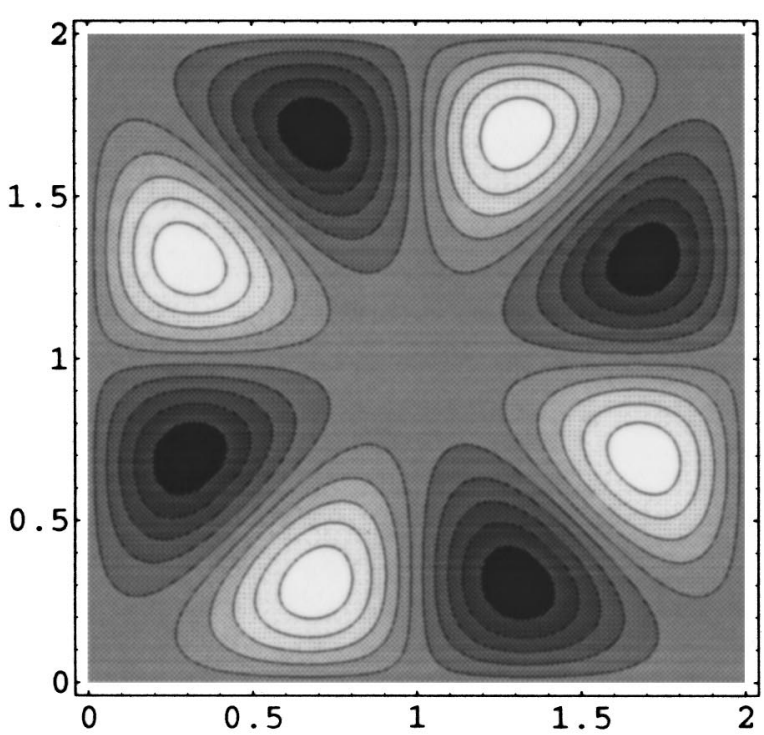

(a)

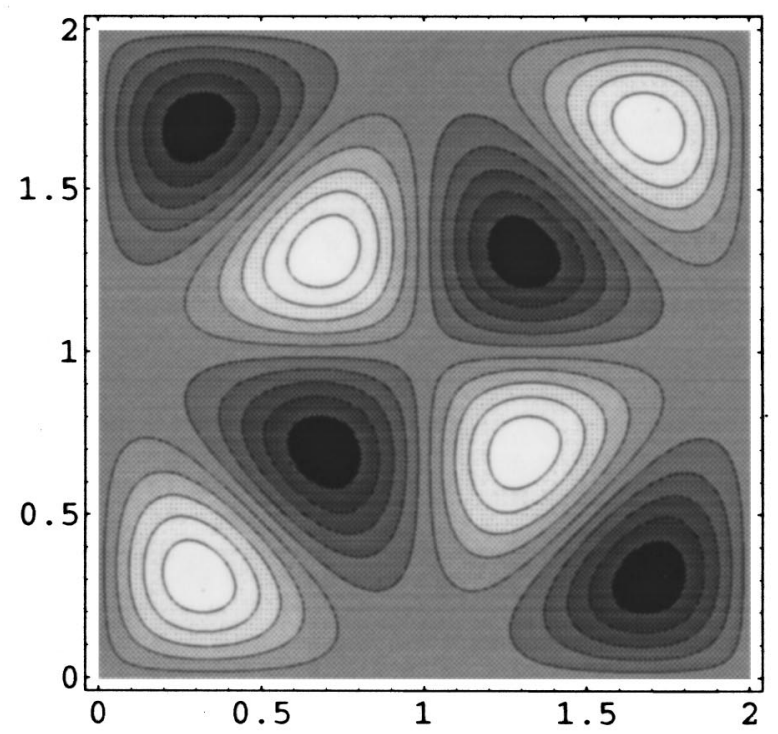

(b)

Fig. 4. Contour plot for the wave function (a) $\phi_{24}^{+}$and (b) $\phi_{24}^{-}$.

above requirements we can avoid dealing with the awkward form of the potential when searching for other symmetries. If a dynamical symmetry operator $\hat{D}$ exists for the particle in the box, it should commute with $\hat{H}$ but not with the $\mathscr{C}_{4 v}$ transformations, since we expect that $\hat{D}$ will mix the $A_{2}$ and $B_{2}$ states (9) as well as the $A_{1}$ and $B_{1}$ eigenfunctions (10), while preserving the boundary conditions satisfied by the $\phi_{n_{1}, n_{2}}^{ \pm}$. These requirements are only possible if $\hat{D}$ transforms one of the two states in (9) or (10) into a linear combination of the same two states. In particular, we shall search for an operator that transforms one of the states into the other. From the point of view of symmetry, this implies that it should have definite tensorial properties under $\mathscr{C}_{4 v}, D^{(\Gamma)}$, where $\Gamma$ is one of the I.R. in Table I, such that
$\Gamma \otimes A_{1}=B_{1}, \quad \Gamma \otimes B_{1}=A_{1}, \quad \Gamma \otimes A_{2}=B_{2}, \quad \Gamma \otimes B_{2}=A_{2}$.

From Table I we readily find that $\Gamma=B_{1}$, since the entries in the table coincide with the group elements for onedimensional representations. Furthermore, from Table I we also see that $B_{1} \otimes E=E$, and thus the degenerate sets with $(-)^{n_{1}} \neq(-)^{n_{2}}$ are left unaltered by a $B_{1}$ tensor. From the right-hand side of Table I we see that in the reference system of Fig. 2 the combination $x^{\prime 2}-y^{\prime 2}$ transforms as the $B_{1}$ I.R. under the group operations, although this is not true in the reference frame chosen for the wave functions (1). The same linear combination of squared momenta, however, transforms according to the $B_{1}$ I.R. in both reference systems. We conclude that the operator

$$
\hat{D}^{\left(B_{1}\right)}=\frac{\partial^{2}}{\partial x^{2}}-\frac{\partial^{2}}{\partial y^{2}},
$$

satisfies the requirements we set up from the outset. As a check, by applying (15) to the states (5) we find

$$
\hat{D}^{\left(B_{1}\right)} \phi_{n_{1} n_{2}}^{ \pm}=\frac{\pi^{2}}{L^{2}}\left(n_{2}^{2}-n_{1}^{2}\right) \phi_{n_{1} n_{2}}^{\mp},
$$

as required. This operator constitutes the "dynamical symmetry" operator (analogous to the Runge-Lenz vector in the Coulomb system), which does not arise from geometrical considerations but from the specific Hamiltonian (12) together with the boundary conditions which impose the $\mathscr{C}_{4 v}$ symmetry. We again stress that in this case the commutation with the Hamiltonian (12) does not automatically guarantee that the boundary conditions are preserved. The combination (15) is the only operator that does so and has the appropriate tensor properties under $\mathscr{C}_{4 v}$. Before considering the combination of this operator with the $\mathscr{C}_{4 v}$ transformations, we remark that it is possible to interpret the larger symmetry in terms of supersymmetric quantum mechanics, in the following sense. ${ }^{9}$ We may identify $\hat{D}^{\left(B_{1}\right)}$ in $(15)$ as a "supersymmetric charge', $\hat{\mathbf{Q}} \equiv \hat{D}^{\left(B_{1}\right)}$, since for the singlet states

$$
\hat{\mathbf{Q}} \phi_{n, n}^{+}=\left(\frac{\partial^{2}}{\partial x^{2}}-\frac{\partial^{2}}{\partial y^{2}}\right) \phi_{n, n}^{+}=0,
$$

and thus each $\phi_{n, n}^{+}$can be considered to be a vacuum state in the language of supersymmetric quantum mechanics (SSQM). ${ }^{8}$ In this approach the $\phi_{n_{1} n_{2}}^{ \pm}$play the role of supersymmetric partners and one may define a supersymmetric Hamiltonian (with $\hat{\mathbf{Q}}^{\dagger}=\hat{\mathbf{Q}}$ ):

$$
\hat{\mathscr{H}}_{s}=\frac{1}{2}\left\{\mathbf{Q}, \mathbf{Q}^{\dagger}\right\}=\left(\frac{\partial^{2}}{\partial x^{2}}-\frac{\partial^{2}}{\partial y^{2}}\right)^{2} .
$$

Since $B_{1} \otimes B_{1}=A_{1}, \hat{\mathscr{H}}_{s}$ commutes with $\mathscr{C}_{4 v}$, and also with both $\hat{H}$ and $\hat{D}^{\left(B_{1}\right)}=\hat{Q}$. Note that the whole spectrum can be organized as a series of "towers" with the singlet $\phi_{n, n}^{+}$, followed by supersymmetric doublets $\phi_{\bar{n}, n}^{ \pm}$, with $\bar{n}>n$. This is an intriguing interpretation, but the description is not complete, since the actual relation between the $\mathscr{C}_{4 v}$ symmetries and $\hat{D}^{\left(B_{1}\right)}$ is not specified in this framework. In Sec. IV we derive a new symmetry group for the system. 


\section{A NEW SYMMETRY GROUP FOR THE} SQUARE WELL

When attempting to define a larger symmetry group for the system which contains the geometrical $\mathscr{C}_{4 v}$ group as a subgroup, we shall need to combine these transformations with the continuous ones generated by $\hat{D}^{\left(B_{1}\right)}$. Finite transformations associated with $\hat{D}^{\left(B_{1}\right)}$ can be obtained by exponentiation:

$$
\hat{U}(\alpha)=\exp \left(i \alpha \hat{D}^{\left(B_{1}\right)}\right)=\exp \left[i \alpha\left(\frac{\partial^{2}}{\partial x^{2}}-\frac{\partial^{2}}{\partial y^{2}}\right)\right],
$$

where $\alpha$ is a coordinate measuring the amplitude of the transformation. Abstractly, the $\hat{U}(\alpha)$ conform to an Abelian one-parameter continuous group, ${ }^{8}$ but no information regarding the range of $\alpha$ can be deduced from (19). When acting with (19) on the square-well wave functions (1) or (5), however, one can see that $\alpha$ is bounded and thus that the group is compact. Using (16) we readily find

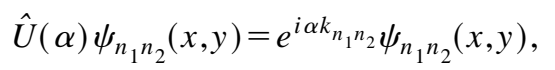

where

$$
k_{n_{1} n_{2}}=\frac{\pi^{2}}{L^{2}}\left(n_{2}^{2}-n_{1}^{2}\right) \text {. }
$$

As $\left(n_{2}^{2}-n_{1}^{2}\right)$ is an integer number, we conclude that $\alpha \pi^{2} / L^{2}$ is a periodic variable, where the period depends on the relative parities of $n_{1}$ and $n_{2}$. Thus, for $(-)^{n_{1}}=(-)^{n_{2}}$,

$$
n_{2}^{2}-n_{1}^{2}=4 l, \quad l \text { integer, }
$$

which implies that we may restrict $\alpha$ to the range

$$
0 \leqslant \frac{\alpha \pi^{2}}{L^{2}} l<\frac{\pi}{2} \quad \text { or } \quad 0 \leqslant \alpha<\frac{L^{2}}{2 \pi l} .
$$

On the other hand, for $(-)^{n_{1}} \neq(-)^{n_{2}}$,

$$
n_{2}^{2}-n_{1}^{2}=2 l+1, \quad l \text { integer, }
$$

implying that

$$
0 \leqslant \frac{\alpha \pi^{2}}{L^{2}}(2 l+1)<2 \pi, \quad \text { or } \quad 0 \leqslant \alpha<\frac{2 L^{2}}{\pi(2 l+1)} .
$$

We see from (20)-(23) that this one-parameter group can be labeled [up to possible additional degeneracies of the "Pythagorean" type (4)] by the discrete set of numbers $k_{n_{1} n_{2}}$ or, alternatively, by the integer numbers

$$
\bar{k}_{n_{1} n_{2}}=\frac{L^{2}}{\pi^{2}} k_{n_{1} n_{2}}=n_{2}^{2}-n_{1}^{2} .
$$

Note that the double-degeneracy (3) implies that $k_{n_{1} n_{2}}$ and $k_{n_{2} n_{1}}=-k_{n_{1} n_{2}}$ are associated with the same energy eigenvalue. The singlet states correspond to $k_{n n} \equiv 0$.

We are now in a position to study the full symmetry operations combining $\hat{U}(\alpha)$ and $\mathscr{C}_{4 v}$. The latter group can be conveniently defined by the coset expansion (see Fig. 2)

$$
\mathscr{C}_{4 v}=\mathscr{C}_{2 v}+\sigma_{d}^{a} \mathscr{C}_{2 v},
$$

where $\mathscr{C}_{2 v}$ is the normal (Abelian) subgroup

$$
\mathscr{C}_{2 v}=\{\hat{g}\}=\left\{E, \sigma_{v}^{a}, \sigma_{v}^{b}, \sigma_{v}^{a} \sigma_{v}^{b}=C_{2}\right\} .
$$

The meaning of (25) is that the full $\mathscr{C}_{4 v}$ group may be generated by considering the subgroup (26) plus the elements arising from the action of $\sigma_{d}^{a}$ on the $\mathscr{C}_{2 v}$ operations. Since the operations $\hat{g} \in \mathscr{C}_{2 v}$ can only change the signs of $\partial x$ and $\partial y$ (see Fig. 2), it is clear from (19) that

$$
\hat{g} \hat{U}(\alpha)=\hat{U}(\alpha) \hat{g},
$$

while $\hat{\sigma}_{d}^{a}$ (which exchanges $\partial x$ and $\partial y$ ) gives

$$
\hat{\sigma}_{d}^{a} \hat{U}(\alpha)=\hat{U}(-\alpha) \hat{\sigma}_{d}^{a} .
$$

We also note that the invariance of $\mathscr{C}_{2 v}$ implies

$$
\hat{\sigma}_{d}^{a} \hat{g}=\hat{g}^{*} \hat{\sigma}_{d}^{a} \quad \text { where } \hat{g}, \hat{g}^{*} \in \mathscr{C}_{2 v},
$$

or explicitly

$$
E^{*}=E, \quad \sigma_{v}^{a^{*}}=\sigma_{v}^{b}, \quad \sigma_{v}^{b^{*}}=\sigma_{v}^{a}, \quad C_{2}^{*}=C_{2} .
$$

Using (27)-(29) we find that in the new symmetry group there are two kinds of elements, which we denote by $\hat{U}(\alpha) \hat{g}$ and $\hat{U}(\alpha) \hat{\sigma}_{d}^{a} \hat{g}$, with multiplication table

$$
\begin{aligned}
& \hat{U}(\alpha) \hat{g}_{1} \hat{U}(\beta) \hat{g}_{2}=\hat{U}(\alpha+\beta) \hat{g}_{1} \hat{g}_{2}, \\
& \hat{U}(\alpha) \hat{g}_{1} \hat{U}(\beta) \hat{\sigma}_{d}^{a} \hat{g}_{2}=\hat{U}(\alpha+\beta) \hat{\sigma}_{d}^{a} \hat{g}_{1}^{*} \hat{g}_{2}, \\
& \hat{U}(\alpha) \hat{\sigma}_{d}^{a} \hat{g}_{1} \hat{U}(\beta) \hat{g}_{2}=\hat{U}(\alpha-\beta) \hat{\sigma}_{d}^{a} \hat{g}_{1} \hat{g}_{2}, \\
& \hat{U}(\alpha) \hat{\sigma}_{d}^{a} g_{1} U(\beta) \hat{\sigma}_{d}^{a} \hat{g}_{2}=\hat{U}(\alpha-\beta) \hat{g}_{1}^{*} \hat{g}_{2} .
\end{aligned}
$$

Denoting the one parameter group of $\hat{U}(\alpha)$ by $D(1)$, it is also clear from (27) and (28) that for $R \in \mathscr{C}_{4 v}$

$$
\hat{R} \hat{U}(\alpha) \hat{R}^{-1}=\hat{U}(\epsilon \alpha),
$$

where

$$
\begin{array}{ll}
\epsilon=+1 & \text { if } R=\hat{g}, \\
\epsilon=-1 & \text { if } \hat{R}=\hat{\sigma}_{d}^{a} \hat{g} .
\end{array}
$$

Equations (32) and (33) imply that under the action of the $\mathscr{C}_{4 v}$ elements, the $D(1)$ set of operators transform among themselves. This is the definition of an invariant subgroup and this indicates that we may denote the symmetry group of (31) in the semidirect product form ${ }^{10}$

$$
\mathfrak{G}=D(1) \wedge \mathscr{C}_{4 v} .
$$

In the next section we construct the I.R. of this group and proceed to prove that under its action the accidental degeneracy (3) is rendered normal.

\section{THE IRREDUCIBLE REPRESENTATIONS OF $\mathfrak{G}$}

Given the semidirect product form of $\mathfrak{G}$, its representations can be constructed using an induction procedure ${ }^{10}$ from $D(1)$ to $\mathfrak{G}$. This is a somewhat technical endeavor which is difficult for the nonspecialist, so in this section we follow a simpler but equivalent route which does not explicitly require the induction concepts. Since we are interested in the particular I.R. of $\mathfrak{G}$ spanned by the square-well's wave functions (1) [or equivalently (5)], we may use the $D(1)$ representations (20) and (21). We start by considering the I.R. of the subgroup of (34) given by

$$
D(1) \otimes \mathscr{C}_{2 v},
$$


Table II. The $\mathscr{C}_{2 v}$ character table.

\begin{tabular}{llrrr}
\hline \hline$C_{2 v}$ & $E$ & $C_{2}$ & $\sigma_{v}^{a}$ & $\sigma_{v}^{b}$ \\
\hline$A_{1}$ & 1 & 1 & 1 & 1 \\
$A_{2}$ & 1 & 1 & -1 & -1 \\
$B_{1}$ & 1 & -1 & 1 & -1 \\
$B_{2}$ & 1 & -1 & -1 & 1 \\
\hline \hline
\end{tabular}

where the direct product sign is due to (27). This is an Abelian subgroup whose I.R. are thus one dimensional. ${ }^{8}$ Denoting the operators of this group by $\hat{U}(\alpha) \hat{g}$, we find

$$
\begin{aligned}
\hat{U}(\alpha) \hat{g} \psi_{n_{1} n_{2}}(\mathbf{x}) & =e^{i \alpha k_{n_{1} n_{2}} \hat{g} \psi_{n_{1} n_{2}}(\mathbf{x})} \\
& =e^{i \alpha k_{n_{1} n_{2}} \chi(\hat{g}) \psi_{n_{1} n_{2}}(\mathbf{x}),}
\end{aligned}
$$

where $\chi(g)$ are elements of the $\mathscr{C}_{2 v}$ character table (see Table II). It is easy to check directly for the $\psi_{n_{1} n_{2}}(\mathbf{x})$ states that

$$
\begin{aligned}
& \chi(E)=1, \quad \chi\left(\sigma_{v}^{a}\right)=(-)^{n_{2}+1}, \quad \chi\left(\sigma_{v}^{b}\right)=(-)^{n_{1}+1}, \\
& \chi\left(C_{2}\right)=(-)^{n_{1}+n_{2}} .
\end{aligned}
$$

Equations (36) and (37) provide a complete classification of the I.R. of the subgroup (35) spanned by the states $\psi_{n_{1} n_{2}}(\mathbf{x})$. Comparing (37) with Table II we readily identify the corresponding $\mathscr{C}_{2 v}$ I.R. In order to generate the corresponding representations for $\mathfrak{G}$, we should now include the action of $\hat{\sigma}_{d}^{a}$ as implied by the coset expansion (25) and the group table (31). Since

$$
\hat{\sigma}_{d}^{a} \psi_{n_{1} n_{2}}(\mathbf{x})=\psi_{n_{2} n_{1}}(\mathbf{x}),
$$

the action of the $\mathscr{C}_{4 v}$ operations that are not in $\mathscr{C}_{2 v}$ span a two-dimensional space whenever $n_{1} \neq n_{2}$. For the case where $n_{1}=n_{2}$, however, the I.R. remains unidimensional:

$$
\hat{U}(\alpha) \hat{R} \psi_{n n}(\mathbf{x})=e^{i \alpha k_{n n}} \chi(R) \psi_{n n}(\mathbf{x})=\chi(R) \psi_{n n}(\mathbf{x}),
$$

for all $R \in \mathscr{C}_{4 v}$, since $k_{n n}=0$. The $\chi(R)$ in (39) are the elements of the $\mathscr{C}_{4 v}$ character table, and we have shown in Eqs.
(9) and (10) that the corresponding I.R. are either $A_{1}$ (for even $n$ ) or $B_{2}$ (for odd $n$ ). The $k_{n n}=0$ I.R. thus coincide with the $\mathscr{C}_{4 v}$ ones. Returning to the $n_{1} \neq n_{2}$ case, we act on the two-dimensional space spanned by $\psi_{n_{1} n_{2}}(\mathbf{x})$ and $\hat{\sigma}_{d}^{a} \psi_{n_{1} n_{2}}(\mathbf{x})=\psi_{n_{2} n_{1}}(\mathbf{x})$,

$$
\begin{aligned}
\hat{U}(\alpha) \hat{g}\left(\begin{array}{c}
\psi_{n_{1} n_{2}} \\
\psi_{n_{2} n_{1}}
\end{array}\right) & =\left(\begin{array}{c}
e^{i k \alpha} \chi(g) \psi_{n_{1} n_{2}} \\
\hat{U}(\alpha) \hat{g} \hat{\sigma}_{d}^{a} \psi_{n_{1} n_{2}}
\end{array}\right) \\
& =\left(\begin{array}{c}
e^{i k \alpha} \chi(g) \psi_{n_{1} n_{2}} \\
\hat{\sigma}_{d}^{a} \hat{U}(-\alpha) \hat{g}^{*} \psi_{n_{1} n_{2}}
\end{array}\right) \\
& =\left(\begin{array}{c}
e^{i k \alpha} \chi(g) \psi_{n_{1} n_{2}} \\
e^{-i k \alpha} \chi\left(g^{*}\right) \hat{\sigma}_{d}^{a} \psi_{n_{1} n_{2}}
\end{array}\right) \\
& =\left(\begin{array}{cc}
e^{i k \alpha} \chi(g) & 0 \\
0 & e^{-i k \alpha} \chi\left(g^{*}\right)
\end{array}\right)\left(\begin{array}{c}
\psi_{n_{1} n_{2}} \\
\psi_{n_{2} n_{1}}
\end{array}\right),
\end{aligned}
$$

where we used (27), (28), and (29) and have written $k_{n_{1} n_{2}}$ as $k$.

Doing the same for $\hat{U}(\alpha) \hat{\sigma}_{d}^{a} \hat{g}$ we find

$$
\hat{U}(\alpha) \hat{\sigma}_{d}^{a} \hat{g}\left(\begin{array}{l}
\psi_{n_{1} n_{2}} \\
\psi_{n_{2} n_{1}}
\end{array}\right)=\left(\begin{array}{cc}
0 & e^{-i k \alpha} \chi(g) \\
e^{i k \alpha} \chi\left(g^{*}\right) & 0
\end{array}\right)\left(\begin{array}{l}
\psi_{n_{1} n_{2}} \\
\psi_{n_{2} n_{1}}
\end{array}\right) .
$$

In both (40) and (41), $\chi(g)$ [or $\left.\chi\left(g^{*}\right)\right]$ are given by (37) [recalling Eqs. (30)]. As a last point in the construction of the I.R., it is necessary to find the representation of $\hat{U}(\alpha) \hat{R}$ in the basis (5), which as discussed before is the one that spans the $\mathscr{C}_{4 v}$ I.R. This is achieved by means of the orthogonal matrix

$$
\mathbf{S}=\frac{1}{\sqrt{2}}\left(\begin{array}{cc}
1 & 1 \\
-1 & 1
\end{array}\right),
$$

which transforms (40) and (41) into the slightly more complicated form

$$
\begin{aligned}
& \mathbf{D}(U(\alpha) g)=\frac{1}{2}\left[\begin{array}{cc}
e^{i k \alpha} \chi(g)+e^{-i k \alpha} \chi\left(g^{*}\right) & e^{-i k \alpha} \chi\left(g^{*}\right)-e^{i k \alpha} \chi(g) \\
e^{-i k \alpha} \chi(g)-e^{i k \alpha} \chi\left(g^{*}\right) & e^{i k \alpha} \chi(g)+e^{-i k \alpha} \chi\left(g^{*}\right)
\end{array}\right], \\
& \mathbf{D}\left(U(\alpha) \sigma_{d}^{a} g\right)=\frac{1}{2}\left[\begin{array}{cc}
e^{i k \alpha} \chi\left(g^{*}\right)+e^{-i k \alpha} \chi(g) & e^{i k \alpha} \chi(g)-e^{i k \alpha} \chi\left(g^{*}\right) \\
e^{i k \alpha} \chi\left(g^{*}\right)-e^{-i k \alpha} \chi(g) & -e^{i k \alpha} \chi\left(g^{*}\right)-e^{-i k \alpha} \chi(g)
\end{array}\right],
\end{aligned}
$$

where $\mathbf{D}$ corresponds to the representation matrix.

Using (43) together with (30) and (37) we can readily find the explicit form of the representation for every $\mathscr{C}_{4 v}$ element, in particular, for the generators $\hat{\sigma}_{v}^{a}$ and $\hat{\sigma}_{d}^{a}$. We can then study the representations of the $\mathscr{C}_{4 v}$ subgroup contained in D, a procedure known as the subduction $\mathfrak{G} \downarrow \mathscr{C}_{4 v}$ by taking $\alpha=0$ in (43):

$$
\mathbf{D}\left(\sigma_{v}^{a}\right)=-\frac{1}{2}\left[\begin{array}{ll}
(-)^{n_{1}}+(-)^{n_{2}} & (-)^{n_{1}}-(-)^{n_{2}} \\
(-)^{n_{1}}-(-)^{n_{2}} & (-)^{n_{1}}+(-)^{n_{2}}
\end{array}\right]
$$

and

$$
\mathbf{D}\left(\sigma_{d}^{a}\right)=\left[\begin{array}{cc}
1 & 0 \\
0 & -1
\end{array}\right] .
$$


From (44) we immediately conclude that when $(-)^{n_{1}}=(-)^{n_{2}}$ the representations are reducible, while for $(-)^{n_{1}} \neq(-)^{n_{2}}$ they remain irreducible, as we had already found in Sec. III.

We have thus succeeded in deriving the explicit form for the I.R. of $\mathfrak{G}$ given in (43), spanned by the square-well states (5) and which are labeled by a pair of quantum numbers $\left(k_{n_{1} n_{2}}, \Gamma\right)$. We should distinguish between the $n_{1}=n_{2}$ and $n_{1} \neq n_{2}$ cases.

(a) For $n_{1}=n_{2}$, all the I.R. are one dimensional, $k_{n n}=0$. The $\Gamma$ is a $\mathscr{C}_{4 v}$ label, either $A_{1}$ or $B_{2}$ for even and odd $n$, respectively.

(b) For $n_{1} \neq n_{2}$, the I.R. of $\mathfrak{G}$ are bidimensional and labeled by $\left(k_{n_{1} n_{2}}, \Gamma\right)$, where $\Gamma$ is a $\mathscr{C}_{2 v}$ I.R. By taking $\alpha=0$ in (43) we arrive at the subduction of these representations $\mathfrak{G} \downarrow \mathscr{C}_{4 v}$. Using the $(\alpha=0)$ characters [the traces of the I.R. associated with (44) and (45)] together with Table I, we find the following results:

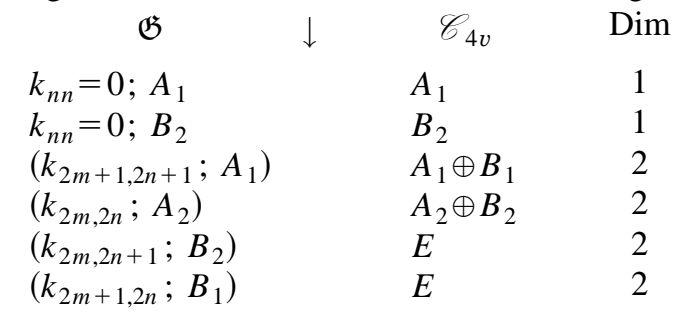

Particular attention should be paid to the third and fourth rows in the above table, corresponding to the I.R. spanned by the basis states (10) and (9). These sets span twodimensional I.R. in $\mathfrak{G}$ which reduce to a direct sum of representations under reduction to $\mathscr{C}_{4 v}$. Therefore, while the degeneracy associated with (9) and (10) is accidental under the geometric $\mathscr{C}_{4 v}$ group, under $\mathfrak{G}$ the degeneracy turns out to be natural. Again, this situation is analogous to the one present in the nonrelativistic hydrogenic atom, where $S O(4)$ and $S O(3)$ play the roles of $\mathfrak{G}$ and $\mathscr{C}_{4 v}$, respectively. The group $\mathfrak{G}$ has a structure similar to that of a space (crystallographic) group, ${ }^{10,11}$ albeit the role of the translations is played by the internal transformations generated by $D^{\left(B_{1}\right)}$, Eq. (19).

\section{CONCLUSIONS}

We have analyzed the problem of a particle enclosed by an impenetrable square box in two dimensions, discussed its accidental degeneracy associated with the existence of a dynamical symmetry, and constructed the higher symmetry group $\mathfrak{G}=D(1) \wedge \mathscr{C}_{4 v}$. The structure of $\mathfrak{G}$ is similar to that of a space group, and we have exploited this fact in order to explicitly construct the I.R. spanned by the square-well wave functions. The doubly degenerate states of the system are now classified according to different double-valued representations of $\mathfrak{G}$, i.e., under the new group the degeneracy has been rendered normal. Furthermore, we have shown that these representations correctly reduce to the appropriate I.R. under the $C_{4 v}$ subgroup.

It should be remarked that our analysis does not explain additional degeneracies [of the "Pythagorean" type (4)] which, as remarked upon in Sec. I, probably require the establishment of a connection between group theory and number theory. ${ }^{7,12}$ Our analysis can be generalized to the three- dimensional free particle system enclosed by an impenetrable cubic box, although the solution is more complex. In this case there is a six-dimensional degeneracy not fully explained by the $O_{h}$ apparent symmetry of the system, and there are two additional dynamical symmetries, which should be combined with the operations of the $O_{h}$ group.

A similar analysis can be applied to other separable systems of the kind

$$
H=\frac{1}{2} P_{x}^{2}+V(|x|)+\frac{1}{2} P_{y}^{2}+V(|y|),
$$

including the free particle and the harmonic oscillator system, both of which display higher symmetries than $\mathfrak{G}$. It is perhaps relevant to note that the quartic Hamiltonian

$$
H=\hat{n}_{x}^{2}+\hat{n}_{y}^{2}
$$

where $\hat{n}_{x}$ and $\hat{n}_{y}$ are the $x$ and $y$ number operators, displays a symmetry group isomorphic to $\mathfrak{G}$ for the space spanned by harmonic oscillator wave functions. In this space the study of the additional degeneracies (4) may be simpler to carry out. ${ }^{3}$ Finally, we note that the same kind of methods can be applied to the analysis of the accidental degeneracies associated with a rectangular box with commensurable sides, that is, where $n L_{1}=m L_{2}$, where $L_{1}$ and $L_{2}$ are the side lengths and $n$ and $m$ are integers. In that case the double degeneracies occur for the levels $\left(n_{1} n_{2}\right)$ and $\left(n_{1}^{\prime}, n_{2}^{\prime}\right)$ when $n n_{1}=m n_{2}^{\prime}$, $m n_{2}=n n_{1}^{\prime}$, besides the appearance of generalized "Pythagorean" identities. Since the patent symmetry in this case is $\mathscr{C}_{2 v}$, no double degeneracies are expected, so the nature of the problem is, in principle, quite different. The integral of the motion (15), however, still connects the degenerate wave functions in the system. In this case the double degeneracy can be ascribed to the presence of a hidden discrete symmetry, which together with the integral of the motion can explain the accidental degeneracy observed. ${ }^{13}$

\section{ACKNOWLEDGMENTS}

This work was supported in part by the European Community under Contract No. CI1*-CT94-0072, DGAPAUNAM under Project Nos. IN105194 and IN105595, CONACyT under Project No. 400340-5-3401E, and Spanish DGICyT under Contract No. PB95-0533-A.

${ }^{1}$ See, e.g., J. M. Jauch and E. L. Hill, "On the problem of degeneracy in quantum mechanics," Phys. Rev. 57, 641-645 (1940); H. V. McIntosh, "Symmetry and Degeneracy," in Group Theoretical Methods and its applications, edited by E. M. Loebl (Academic, New York, 1990), Vol. II, pp. 75-137; M. Moshinsky, “Accidental Degeneracies and Symmetry Groups,' Found. Phys. 13, 73-79 (1983).

${ }^{2}$ L. Hulthen, "Über die Quanten Mechanische Herleitung der Balmerterme,', Z. Phys. 86, 21-23 (1933); V. Fock, “'Zur Theorie des Wasserstoffatoms,' ibid. 98, 145-154 (1936); M. J. Englefield, Group Theory and the Coulomb Problem (Wiley Interscience, New York, 1972); X. L. Yang, M. Lieber, and F. T. Chan, "The Runge Lenz Vector for the Twodimensional Hydrogen Atom,', Am. J. Phys. 59, 231-232 (1991).

${ }^{3}$ M. Moshinsky, C. Quesne, and G. Loyola, "Art of Science: The determination of the symmetry Lie algebra for a Hamiltonian with accidental degeneracy," Ann. Phys. 198, 103-131 (1990).

${ }^{4}$ See, e.g., V. I. Man'ko, "Invariants and States Generating Symmetry of Nonstationary Systems," in "Symmetries in Science, edited by B. Gruber, L. C. Biedenharn, and H. D. Doebner (Plenum, New York, 1991), Vol. V, pp. 453-473.

${ }^{5}$ R. L. Liboff, Introductory Quantum Mechanics (Holden-Day), pp. 297298, San Francisco, California, 1980.

${ }^{6}$ See, e.g., C. Cohen-Tannoudji, Quantum Mechanics, (Wiley Interscience, New York, 1977), Vol. I. 
${ }^{7} \mathrm{~W} .-\mathrm{K} . \mathrm{Li}$, "Degeneracy in the particle-in-the-square problem," Am. J. Phys. 50, 666 (1982).

${ }^{8}$ M. Hamermesh, Group Theory and its Application to Physical Problems (Dover, New York, 1962).

${ }^{9}$ R. W. Haymaker and R. P. Rau, "Supersymmetry in Quantum Mechanics," Am. J. Phys. 54, 928-936 (1986); F. Cooper and B. Freedman, "Aspects of Supersymmetric Quantum Mechanics," Ann. Phys. 146, 262288 (1983); M. de Crombrugghe and V. Rittenberg, "Supersymmetric Quantum Mechanics,"' ibid. 151, 99-126 (1983).
S. L. Altmann, Induced Representations in Crystals and Molecules (Academic, New York, 1977).

${ }^{11}$ W. Ludwig and C. Falter, Symmetries in Physics, Group Theory Applied to Physical Problems, Springer Series in Solid-State Sciences Vol. 64 (Springer, New York, 1988).

${ }^{12}$ E. B. Bogomolny, B. Georgeot, M. J. Giannoni, and C. Schmith, "Chaotic Billiards Generated by Arithmetic Groups," Phys. Rev. Lett. 69, 14771480 (1992).

${ }^{13}$ A. Frank, R. Lemus, M. Andres, and F. Leyvraz (unpublished). 